\title{
Pseudobankesia lichenaria spec. nov., eine neue Psychiden-Art aus der Zagoria im griechischen Voria Pindos-Gebirge (Lepidoptera: Psychidae)
}

\author{
Mit 5 Figuren, 1 Karte und 1 Tabelle
}

Michael WeIDLICH ${ }^{1}$

${ }^{1}$ Lindenallee 11, 15898 Neißemünde, OT Ratzdorf, Deutschland. - dr.michael.weidlich@gmail.com Published on 2016-06-30

\section{Zusammenfassung}

Es wird Pseudobankesia lichenaria spec. nov. aus der Zagoria beschrieben. Die Art wurde bisher nur an einer Stelle bei Kipi beobachtet. Angaben zur Biologie und Ökologie werden mitgeteilt, wonach die neue Art sehr versteckt in Hohlräumen an Felswänden lebt. Weiterhin wird ein Überblick der bereits bekannten Pseudobankesia-Arten auf dem Balkan gegeben.

\section{Key words}

Greece, Voria Pindos, Zagoria, Pseudobankesia lichenaria spec. nov., Psychidae, Lepidoptera

\section{Summary}

Pseudobankesia spec. nov. from the Zagoria is described. Informations on their biology and ecology are reported. The species live very hidden in cracks at rocks. Furthermore, an overview on the Pseudobankesia species distributed to the Balkans is given.

\section{Einleitung}

Seit vielen Jahren beschäftigt sich der Autor mit der Psychidenfauna des Balkans, insbesondere der von Griechenland. Die ersten Ergebnisse sind bereits veröffentlicht worden, worunter sich auch die Erstbeschreibungen von Dahlica thessaliensis, D. zagoriensis, D. pangeoensis und Epichnopterix hellenidensis befinden (vergl. WEIDLICH, 1989, 2008, 2012 a, b, c, 2013, a, b, c, d, 2014). Auf dem Balkan kommen noch weitere, bisher unbeschriebene Pseudobankesia-Arten vor, darauf ist bereits hingewiesen worden (WEIDLICH, 2008: 477, 499 und WEIDLICH, 2013d: 329).
In der vorliegenden Arbeit wird nun eine weitere Pseudobankesia-Art beschrieben, die im Jahre 2003 entdeckt und auch in den Jahren 2005, 2006, 2007, 2008, 2010, 2012 und 2015 beobachtet wurde. Ihre sehr charakteristischen, auf dem Balkan einzigartigen Säcke wurden bisher nur in einem Habitat in der Zagoria gefunden. Die anschließenden Zuchten erwiesen sich trotz umfangreichem Material als langwierig und sehr schwierig.

Auf die Kenntnisse zur Schmetterlingsfauna der Zagoria wurde bereits in einer anderen Arbeit vom Verfasser verwiesen (WEIDLICH, 2012c). 


\section{Beschreibung der Pseudobankesia} lichenaria spec. nov.

\section{Pseudobankesia lichenaria spec. nov.}

Derivatio nominis: Die neue Art wird nach den sehr charakteristischen Säcken, die nahezu ausschließlich mit Flechtenteilen belegt sind, benannt.
Holotypus: $\sigma^{\top}$, e.l. Juni 2008, Europa meridionalis, Greece, Voria Pindos, Zagoria, Kipi $1 \mathrm{~km}$ SW, $750 \mathrm{~m} \mathrm{NN}$, leg. Dr. M. Weiduich (Fig. 1). Er befindet sich in coll. Museum für Naturkunde des Leibniz Institutes in Berlin.

\section{Paratypen (die gezüchteten Exemplare jeweils mit Sack und Puppenhülle):}

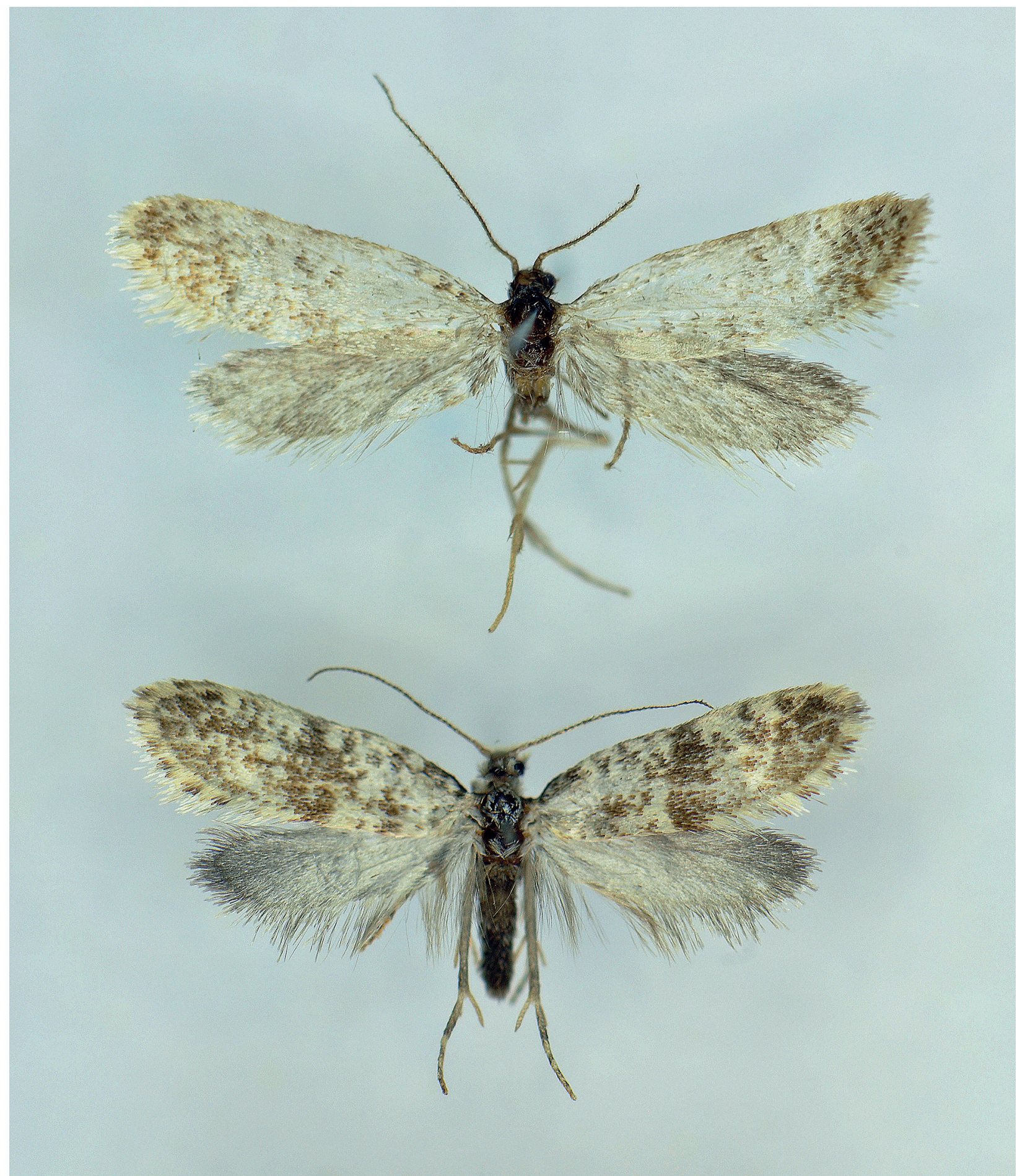

Fig. 1: Pseudobankesia lichenaria spec. nov., Holotypus: đo e.l. Juni 2008 (oben, Flügelspanne 11,8 mm), Paratypus, e.l. 14.06.2015 (unten): Europa meridionalis, Greece, Voria Pindos, Zagoria, Kipi 1 km SW, $750 \mathrm{~m} \mathrm{NN}$, alles leg. Dr. M. WeIdLICH. (Foto: M. WeIDLICH). 
Männchen: $4 \sigma^{\star} \sigma^{\star}$, e.l. Juli 2007, $1 \sigma^{\star}$, e.l. Juni 2008, $1 \sigma^{\star}$, e.l. Juni 2010, $1 \sigma^{\top}$, e.l. 14. Juni 2015: Europa meridionalis, Greece, Voria Pindos, Zagoria, Kipi 1 km SW, 750 m NN, leg. Dr. M. WeIDLICH.

Weibchen: 1 \& , e.l. 01.07.2015, Europa meridionalis, Greece, Voria Pindos, Zagoria, Kipi 1 km SW, 750 m NN, leg. Dr. M. Weidlich.

Säcke: 266 Säcke 09.-10.05.2003, Europa meridionalis, Greece, Voria Pindos, Zagoria, Kipi $1 \mathrm{~km}$ SW, $750-800$ m NN; 103 Säcke 10.04.2005; 36 Säcke 05.-06.04.2006; 64 Säcke 20.04.2007; 289 Säcke 09.-10. 04.2008; 101 Säcke 17.04.2010; 96 Säcke 18.04. 2012; 128 Säcke 03.05.2015: Europa meridionalis, Greece, Voria Pindos, Zagoria, Kipi $1 \mathrm{~km} \mathrm{SW,} 750 \mathrm{~m} \mathrm{NN}$, alles leg. Dr. M. WeIDLICH.

Raupen: 1 Raupe 05.-06.04.2006, 5 Raupen 09.-10.04. 2008, 8 Raupen 22.05.2015: Europa meridionalis, Greece, Voria Pindos, Zagoria, Kipi $1 \mathrm{~km} \mathrm{SW,} 750 \mathrm{~m} \mathrm{NN}$, alles leg. Dr. M. WeIDLICH.

Das vorliegende Typenmaterial, welches in 8 Jahren eingetragen wurde, umfasst 8 Männchen mit Säcken, 1 Weibchen mit Sack, 14 adulte Raupen und weitere 1.083 Säcke, darunter 3 mit männlicher und 3 mit weiblicher Puppenhülle. Es befindet sich in coll. Museum für Naturkunde des Leibniz Institutes in Berlin, coll. W. ArNsCheid (Bochum) und coll. M. WeIDLICH.

Diagnose: Männchen: kleine Falter mit einer Flügelspanne von 9,6 bis $11,8 \mathrm{~mm}$, Augen schwarz, rund, mit Ocellen. Labialpalpen dreigliederig. Der Augenindex (Quotient aus Augenabstand zum maximalen Augendurchmesser) beträgt rund 1,3. Stirnschopfbehaarung lang silbriggrau. Fühler sehr lang; sie erreichen in ihrer Länge deutlich mehr als die Hälfte des Vorderflügelcostalrandes. Sie sind beschuppt, die Bewimperung ist einseitig ventral angeordnet und ihre Länge entspricht etwa der Fühlergliedlänge (Bereich des 10. bis 15. Fühlergliedes). Fühlergliederzahl mit Scapus und Pedicellus 34 bis 35. Körper grau beschuppt.

Vorderflügelfärbung silbrig mit bräunlicher, unregelmäßig verteilter Punktierung. Diskoidalfleck nicht erkennbar. Vorderflügel dicht beschuppt mit breiten, vier- bis sechszackigen Deckschuppen (Schuppenklasse IV-V, nach SAUTER, 1956); Fransenschuppen ebenfalls breit, meist vier- bis fünfzackig, untergeordnet zwei- bis dreizackig. Aderung ohne Entschuppung nicht erkennbar. Vorderflügeladerung mit Anhangszelle und mit 10 Diskoidalzelladern. Radius 2 und 3 entspringen aus einem Punkt, Radius 4 und 5 sind sehr lang gestiehlt. Hinterflügelfärbung grau, die Fransen etwas heller, Aderung kaum erkennbar, ohne Eingeschobene Zelle und mit 6 Diskoidalzelladern, die alle getrennt voneinander entspringen.

Vordertibien mit Epiphyse, Mitteltibien mit einem Spornpaar, Hintertibien mit zwei Spornpaaren. Alle
Beine mit 5 Tarsengliedern. Genitalapparat Pseudobankesia - typisch, Tegumendach eingekerbt mit zwei Spitzen, Saccus kurz und breit. Phallus schwach gebogen, ohne Cornuti und Stützstab (vergl. Fig. 2).

Weibchen: Flügellos, Gesamtfärbung hellgelblich, 2,4 mm lang (ohne Ovipositor) und 0,8 $\mathrm{mm}$ im Durchmesser. Augen klein, oval und schwarz, keine zusätzlichen Ocellen. Fühler relativ kurz mit 12 Fühlergliedern (mit Scapus und Pedicellus). Körper gestreckt und schütter hell behaart. Das 7. Abdominalsegment ringförmig behaart. Die Behaarung ist sehr dicht und von gelblichweißer Färbung. An den Vorder- und Hinterbeinen 3, an den Mittelbeinen 4 Tarsenglieder. Weiterhin an der Hinterbeinen ein kurzes, in der Form breites Spornpaar. Bei der Kopf-Brustplatte der Puppe sind die Fühlerscheiden deutlich länger als die der Beinscheiden.

Raupe: Länge 2,9 bis $3,1 \mathrm{~mm}$ und Breite um 0,9 bis $1,1 \mathrm{~mm}$. Kopf und erstes Thorakalsegment dunkelbraun, stark sklerotisiert ohne Zeichnungselemente. 2. und 3. Thorakalsegment braun und schwächer sklerotisiert. Körper gelblich bis hellbraun. An der ventralen Seite des 3. bis 6. Abdominalsegmentes befinden sich ovale Ringstrukturen, die aus kleinen, braunen chitinisierten Flecken bestehen. Auch auf dem letzten, dem 9. Abdominalsegment befinden sich derartige Strukturen. Diese sind alle paarig angeordnet. Letztes Abdominalsegment ebenfalls braun und stärker sklerotisiert.

Sack: relativ groß, 6,5 bis $8,8 \mathrm{~mm}$ lang und 3 bis 5 breit, deutlich abgeflacht und um $2 \mathrm{~mm}$ in der Höhe messend. Die Säcke sind meist zweikantig, die dorsale Kante nicht vorhanden oder sehr schwach ausgeprägt. Ihre Färbung ist gelblich- grünbläulich (frische Säcke!) und die Säcke bestehen aus versponnenen Flechtenpartikeln (Fig. 3 und 4).

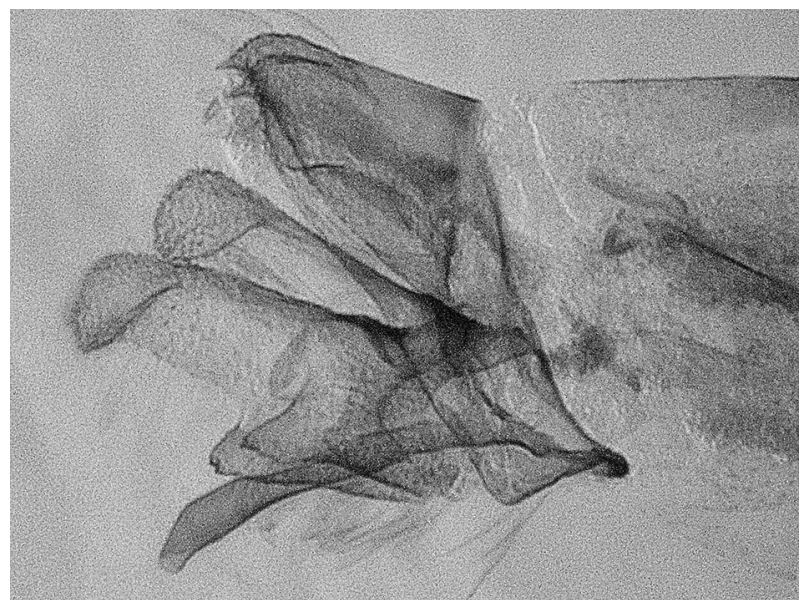

Fig. 2: P. lichenaria spec. nov., Holotypus: ${ }^{\star}$, Genital, Lateralansicht: Europa meridionalis, Greece, Voria Pindos, Zagoria, Kipi $1 \mathrm{~km} \mathrm{SW}, 750 \mathrm{~m} \mathrm{NN}$, leg. Dr. M. Weidulch. (Foto: W. R. ARNSCHEID). 


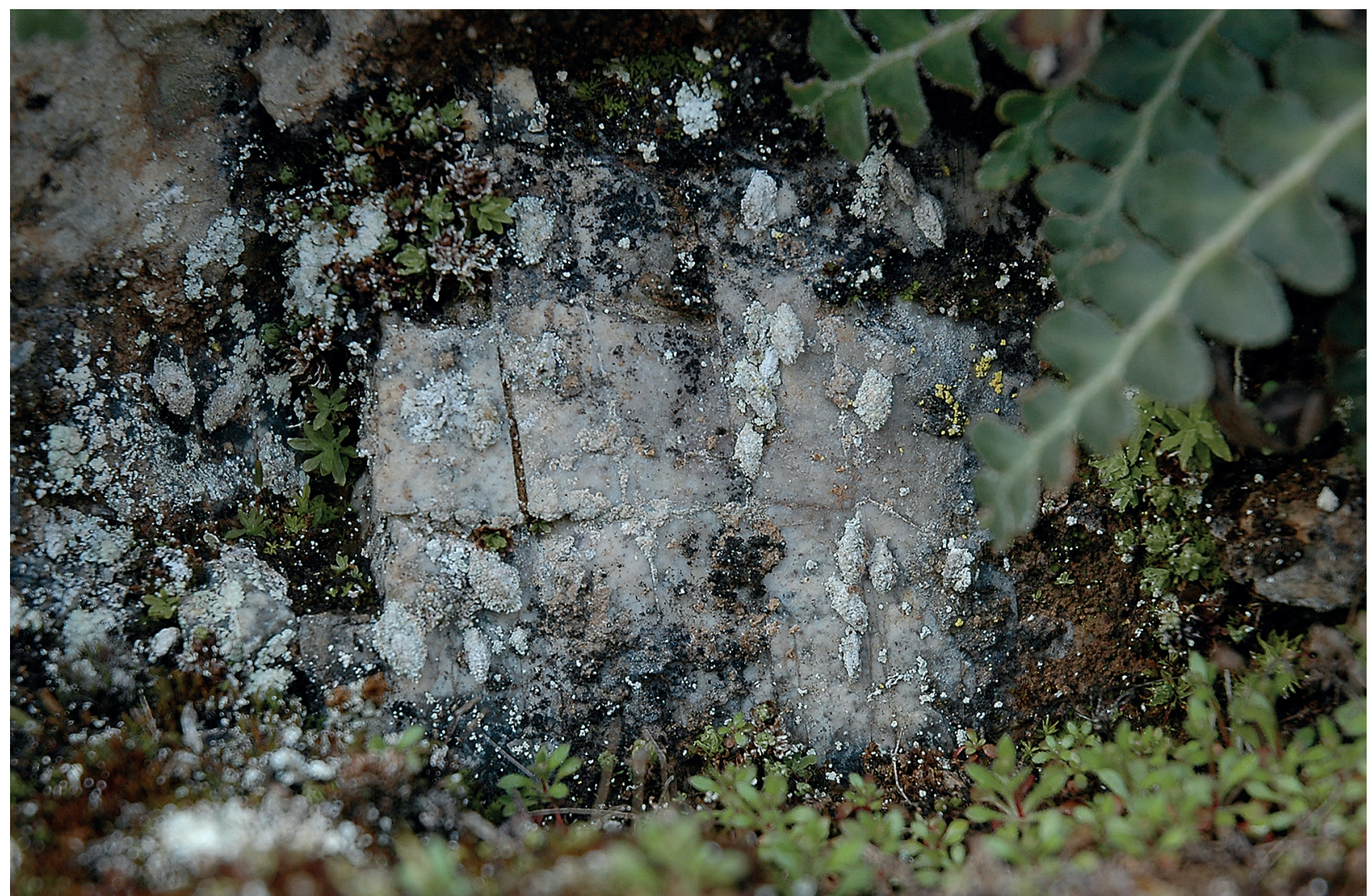

Fig. 3: Eine Ansammlung von Pseudobankesia lichenaria spec. nov. - Säcke in einer Felsnische: Kipi 1 km SW, 750 m NN. (Foto: M. WeIDLICH, 10.04.2008).

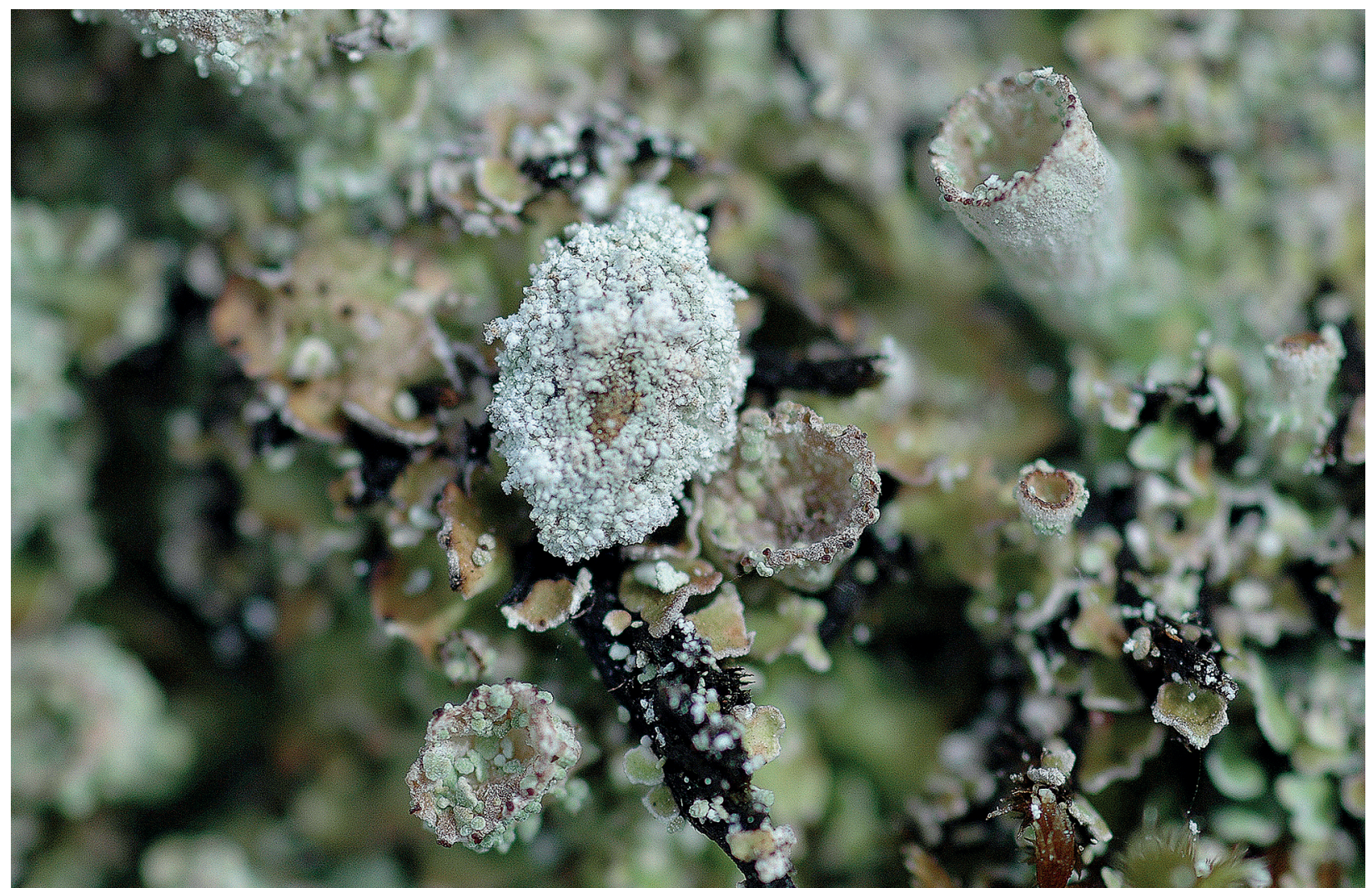

Fig. 4: Detailfoto von einem P. lichenaria spec. nov. - Sack in der Flechtengesellschaft: Kipi 1 km SW, 750 m NN. (Foto: M. WeIDLICH, 10.04.2008). 
Differentialdiagnose: P. lichenaria spec. nov. ist durch wesentliche Merkmale der Gattung Pseudobankesia MeIER, 1963 charakterisiert. Vom Balkan sind bisher 5 hier endemische Arten dieser Gattung beschrieben worden. In Griechenland kommen drei Arten vor, je eine weitere Art in Griechenland und Mazedonien sowie in Bulgarien (Karte 1). Von Zypern ist noch eine weitere, ebenfalls endemische Art, bekannt.

P. lichenaria spec. nov. wird mit den oben genannten sechs Arten verglichen. In der Größe unterscheiden sich die Pseudobankesia-Arten des Balkans und Zyperns kaum voneinander. In der Vorderflügelfärbung ähnelt die neue Art am meisten $P$. arahova STENGEL, 1990 und $P$. darwinii STENGEL, 1990. Auffallend gering fällt der Augenindex mit 1,3 aus, der bei den anderen Balkanarten zwischen 1,5 bis fast 2 liegt. Charakteristisch sind ebenfalls die sehr langen Fühler mit 34 bis 35 Gliedern.

Die Vorderflügeladerung der neuen Art zeigt ebenfalls einige spezifische Merkmale auf: Radius 2 und 3 entspringen einem Punkt und Radius 4 und 5 sind sehr lang gestiehlt, wie es von keiner anderen PseudobankesiaArt sowie auch bei $P$. aphroditae bisher bekannt ist.

Die graue Hinterflügelfärbung tritt in dieser Ausprägung nur bei P. kresnensis WeIDLICH, 2014 auf.
Unterschiede lassen sich auch bei den Weibchen nachweisen. Die Kombination von 3 und 4 Tarsengliedern wurde bisher bei keiner anderen Pseudobankesia-Art des Balkans studiert.

Die Säcke von P. lichenaria spec. nov. ähneln denen von P. hauseriella und P. aphroditae sowie den mittel- und westeuropäisch verbreiteten $P$. alpestrella (HEINEMANn, 1870), P. vernella (Constant, 1899) und P. contractella HäTTENSCHWILER, 1994 und bestehen hauptsächlich aus Flechtenpartikeln. Die Form dieser 5 genannten Arten ist im Querschnitt auffallend dreikantig. Die größten Ähnlichkeiten bezüglich des verwendeten Sackmaterials (Flechten), sowie in ihrer plattgedrückten Form und kaum erkennbarer Dorsalkante (zweikantig) bestehen zu P. casaella HäTtensChWILER (1994), deren Verbreitung in Andorra, Spanien und Frankreich liegt (ARNSCHEID, 2014: 344-345).

Weitere, wichtige Unterscheidungsmerkmale sind in der Tabelle 1 dargestellt.

Biologie und Ökologie: P. lichenaria spec. nov. ist univoltin und die Säcke wurden zwischen dem 05.04. und 10.05., hauptsächlich jedoch im April, gesammelt. Die Zucht der eingetragenen Raupen erwies sich in jedem Jahr als schwierig und war sehr verlustreich. Obwohl die

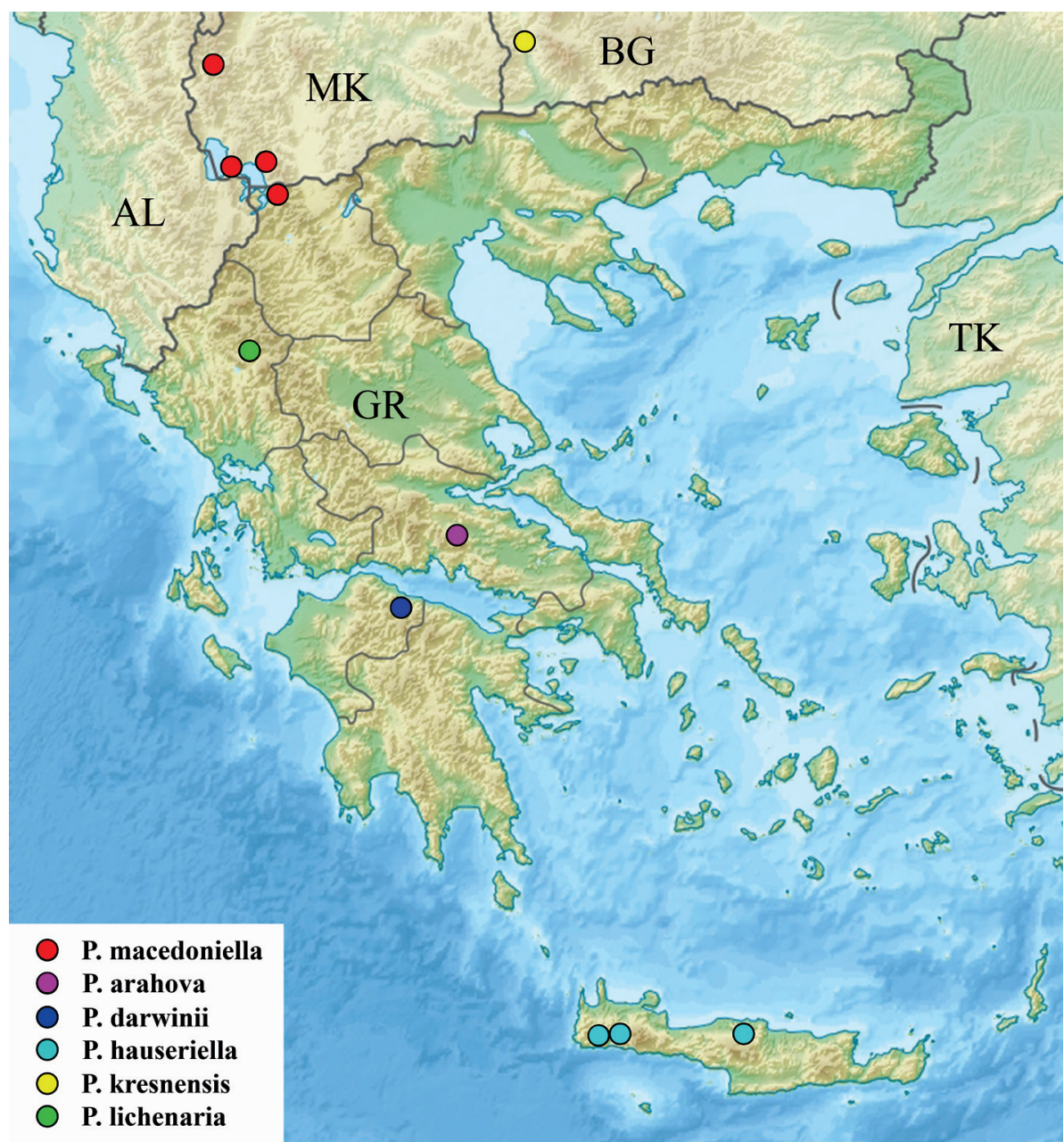

Karte 1: Die Verbreitung der Pseudobankesia-Arten auf dem Balkan (verändert nach https:// de.wikipedia.org/wiki/Griechenland\#/media/File:Greece_relief_location_map.jpg). 


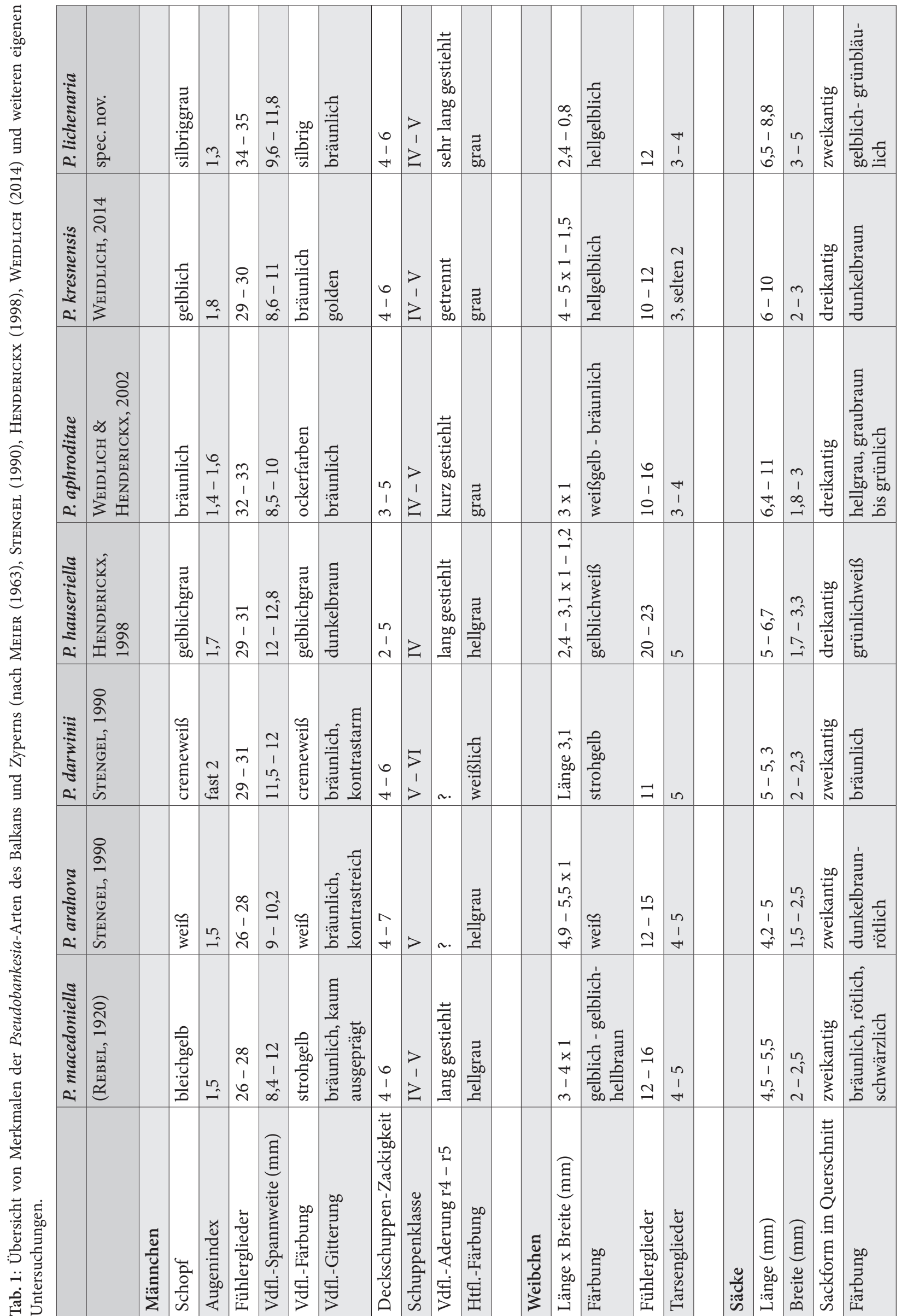




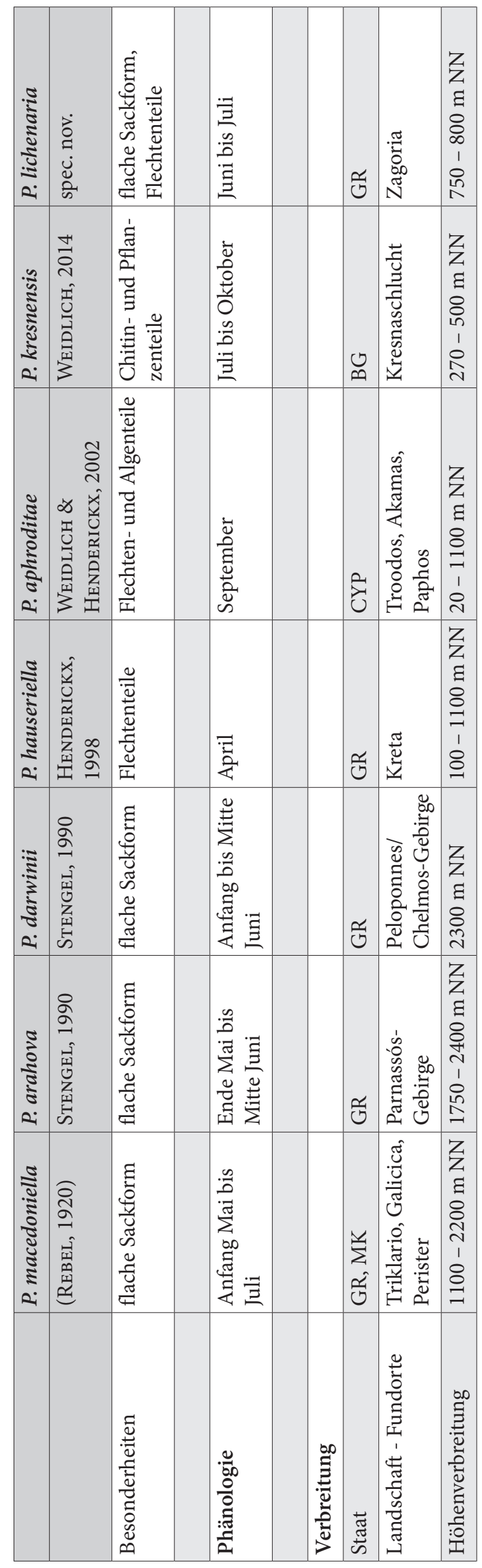

Habitatbedingungen dabei weitgehend nachempfunden wurden, die Tiere auf mehrere, verschiedene Zuchtbehälter verteilt wurden, ging ein Großteil der Zuchten ein. Alle Imagines schlüpften im Juni und Juli.

Weiterhin konnte in einigen Fällen der Bau eines Behelfssackes beobachtet werden. Wenn beträchtliche Teile des Sackes durch andere Raupen von P. lichenaria spec. nov. gefressen bzw. zerstört wurden, fertigten die Raupen aus den Resten und umliegenden Material einen neuen Sack, der nun andersfarbig (bräunlich) und eine röhrenartige Form aufwies. Es bestehen nunmehr keine Ähnlichkeiten mehr zu dem arttypischen Sack. Die Besonderheit zur Anfertigung von Behelfssäcken wurde bereits von WeIdlich (2008: 476) für den Holotypus von Dahlica pseudoachajensis STENGEL, 1990 diskutiert.

Den Lebensraum bei Kipi bilden flechten- und moosreiche, schattige und im Frühjahr feuchte Nischen in Felswänden. Die besiedelten Felsbereiche befinden sich in Höhenlagen zwischen 750 und $800 \mathrm{~m} \mathrm{NN}$, sind nach Westen ausgerichtet und werden zusätzlich beschattet von Vertretern der Strauchschicht (z. B. Ostrya carpinifolia Scop., (siehe Fig. 5).

$P$. lichenaria spec. nov. ist vergesellschaftet mit folgenden Psychidenarten, die aber mehr trockene und exponiertere Bereiche im selben Habitat besiedeln: Eumasia parietariella (Heydenreich, 1851), Dahlica zagoriensis WEIDLICH, 2012, Psyche crassiorella (BRUAND, 1850), Epichnopterix hellenidensis WeIDLICH, 2013, Reisseronia spec., Canephora hirsuta (PodA, 1761), Pachythelia villosella (OCHSENHEIMER, 1810) und Apterona helicoidella (VALLOT, 1827).

\section{Danksagung}

Herzlich gedankt sei meinen Freund W. R. Arnscheid (Bochum) für die Anfertigung des Genitalpräparates und des Fotos und für seine kritische Durchsicht des Manuskriptes.

\section{Literatur}

Arnscheid, W. R. 2014: Distribution et écologie de Penestoglossa pyrenaella Herrmann, 2006 et de Pseudobankesia casaella Hättenschwiler, 1994 en France et dans la Péninsule ibérique (Lepidoptera Psychidae). - L'Entomologiste 70 (6): 341-345.

HäTtenschwileR, P. 1994: Die mittel- und westeuropäischen Arten der Gattung Pseudobankesia mit Beschreibung von zwei neuen Arten (Lep. Psychidae). - Mitteilungen Entomologischen Gesellschaft Basel 44 (3/4): 109-121.

Henderickx, H. 1998: A new Pseudobankesia species from Crete (Lepidoptera: Psychidae). - Phegea 26 (1): $1-8$. 
Meier, H. G. 1963: Zur Kenntnis der Gattungen Pseudobankesia gen. nov. und Bankesia Tutr (Lep., Psychidae) (5. Beitrag zur Kenntnis der Psychiden). - Mitteilungen der Münchener Entomologischen Gesellschaft 53: 1-23.

Stengel, D. 1990: Drei neue Psychidae-Arten aus Griechenland und ein neuer Status für Peleponnesia glaphyrella culminella SIEder (Lepidoptera, Psychidae). - Atalanta 20: 211-217 (1989).

Weidlich, M. 1989: Abriß der Psychidenfauna Bulgarisch-Mazedoniens mit der Erstbeschreibung des Weibchens und des Sackes von Reisseronia nigrociliella (ReBel, 1934) (Lepidoptera, Psychidae). - Nachrichtenblatt der Bayerischen Entomologen 38 (1): 1-12.

Weidlich, M. 2008: Beitrag zur Lepidopteren-Fauna des Notia Pindos (Tringia-Massiv, Lákmos-Gebirge und Athamáno-Gebirge) in Griechenland mit Beschreibung von zwei neuen Arten sowie Angaben zur Köcherfliegen- (Trichoptera) und Schnakenfauna (Diptera: Tipulidae). - Entomofauna 29 (27): 469-504.

Weidlich, M. 2012a: Zur Kenntnis von Loebelia crassicornis (STAUDINGER, 1871) (Lepidoptera: Psychidae). - Entomologische Zeitschrift $122(2)$ : 79-85.

WeIDLICH, M. 2012b: Zur Verbreitung und zur Biologie des Balkanendemiten Stichobasis helicinoides (Heylaerts, 1879) (Lepidoptera: Psychidae). Microlepidoptera.hu 5: 43-48.
WeIDLICH, M. 2012c: Zur Schmetterlings-Fauna der Zagoria im Voria Pindos (Griechenland) mit der Beschreibung einer neuen Psychidenart (Lepidoptera, Psychidae). - Linzer biologische Beiträge $44(2)$ : 1237-1262.

Weidlich, M. 2013a: Eine neue Psychide aus den Helleniden Griechenlands und Albaniens (Lepidoptera: Psychidae). - Nachrichten des entomologischen Vereins Apollo N.F. 33 (4): 145-148.

WeIDLICH, M. 2013b: Zur Verbreitung des Balkanendemiten Pseudobankesia macedoniella (REBEL, 1920) (Lepidoptera: Psychidae). - Microlepidoptera. hu 6: 23-27.

Weidlich, M. 2013c: Zur Schmetterlings-Fauna des Pangéo-Gebirges in Griechisch-Mazedonien mit Beschreibung von Dahlica pangeoensis nov. sp. und Bemerkungen zur Köcherfliegenfauna (Lepidoptera, Trichoptera). - Linzer biologische Beiträge 45 (1): 945-969.

Weidlich, M. 2013d: Zur Psychidenfauna Albaniens unter Berücksichtigung der Albanien-Expedition 1961 des Deutschen Entomologischen Institutes. Beiträge zur Entomologie 63 (2): 325-338.

WeIDlich, M. 2014: Pseudobankesia kresnensis nov. spec., eine neue Psychiden-Art aus Bulgarisch-Mazedonien (Lepidoptera: Psychidae). - Entomologische Nachrichten und Berichte 58 (1/2): 21-25.

Weidlich, M. \& Henderickx, H. 2002: Eine neue Pseudobankesia aus Zypern (Lep. Psychidae). - Linzer biolologische Beiträge 34 (1): 503-512.

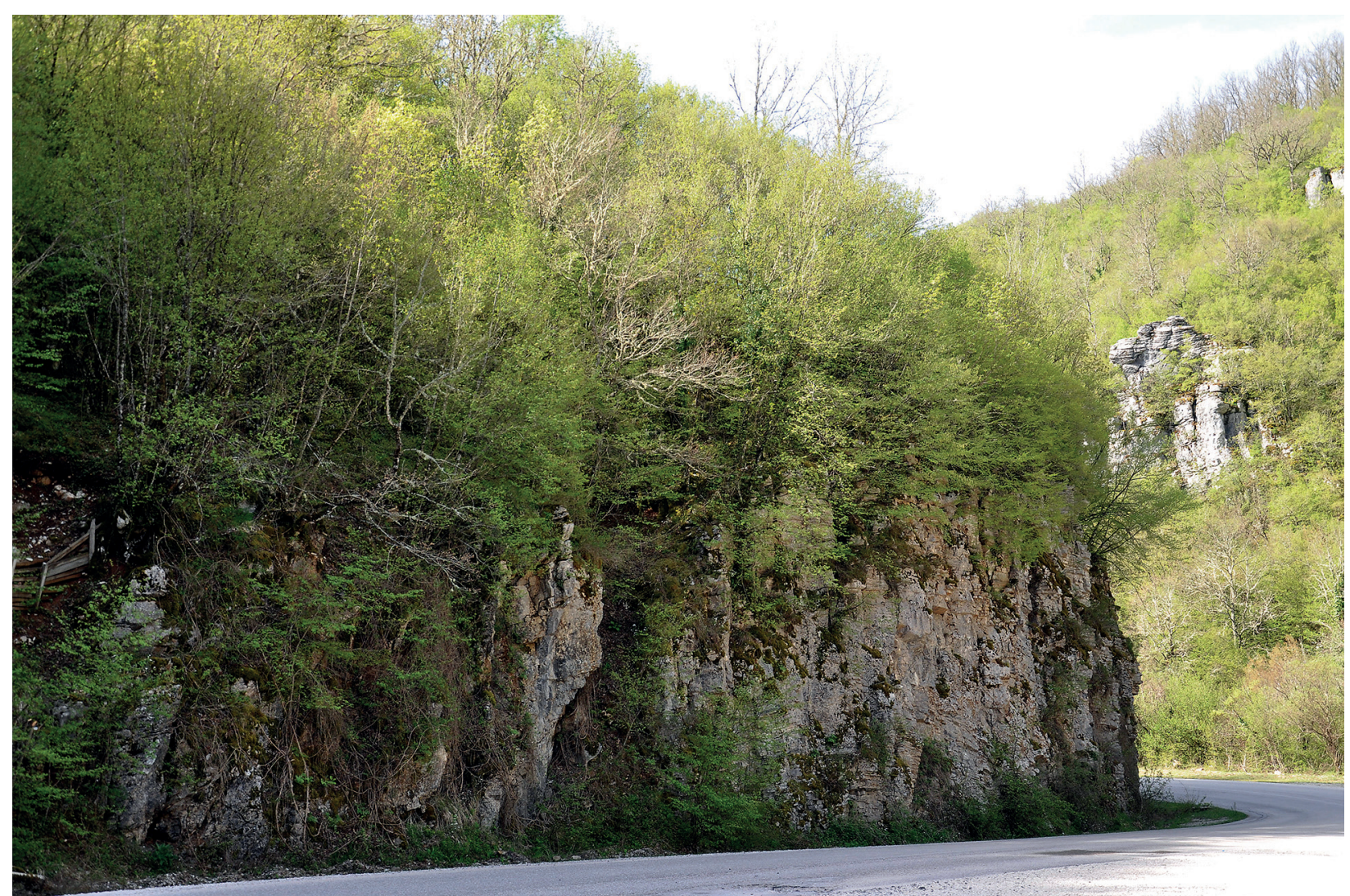

Fig. 5: Lebensraum von P. lichenaria spec. nov.: Kipi 1 km SW, 750 m NN. (Foto: M. WeidLICH, 03.05.2015). 